\title{
LA EDUCACIÓN TÉCNICA EN CHILE Y ESTADOS UNIDOS DESDE UNA PERSPECTIVA HISTÓRICA Y COMPARADA
}

\section{Introducción}

Estados Unidos y Chile optan, hoy en día, por diferentes estrategias de provisión de educación técnica en el sistema escolar. Mientras que en el país del norte se impone un modelo comprehensivo que elude la distinción formal entre planes de estudios técnicos y académicos de nivel medio, en Chile prima un modelo segmentado en el que ambas opciones formativas están claramente definidas y se imparten de manera separada, ya sea al interior de una misma unidad educacional, o bien en establecimientos especializados. Sin embargo, en sus orígenes, las características de este tipo de educación fueron similares en ambos países, en el sentido de que las primeras escuelas técnicas nacen focalizadas en sectores económicos determinados y desligadas de la educación académica.

¿Por qué la educación técnica evoluciona de distinto modo en los sistemas escolares? ¿Qué factores o medidas determinan el surgimiento de ciertos modelos en reemplazo de otros? ¿Son razones económicas, sociopolíticas o ideológicas las que están detrás de las reformas que afectan y definen a la educación técnica? Este artículo revisa los principales sucesos que caracterizaron la evolución de la educación técnica, tanto en Estados Unidos como en Chile, desde una mirada histórica y comparada. El interés está en identificar los distintos enfoques de aproximación a la educación técnica que influyen en los hitos trascendentes de este tipo de educación en ambos contextos. Con ello se busca abrir el debate respecto de la educación técnica en el sistema escolar chileno, y su relación con la vertiente académica y la educación superior.

Múltiples autores (Benavot, 1983; Lakes, 1997; HyslopMargison, 2000; Rojewski, 2009) dan cuenta de al menos tres 
perspectivas de conceptualización de la educación técnica. La primera y más común es la que proviene de la teoría técnico-funcionalista de capital humano y concibe a esta opción formativa como una educación especializada, en función de los requerimientos del aparato productivo y el progreso económico. Según esta visión, la educación técnica tiene como misión principal alcanzar la eficiencia y el orden social a través de prescripciones curriculares que preparen a los individuos específicamente para el papel que desempeñarán en los mercados laborales, en concordancia con sus capacidades y aptitudes. Se impone la idea, entonces, de que el éxito o fracaso ocupacional es el resultado del mérito y esfuerzo individual y no de la estructura de oportunidades (Kantor, 1986).

Una segunda perspectiva de acercamiento a la educación técnica es la que proviene de los llamados "argumentos integracionistas" que abogan por una formación que prepare para los desafíos de la vida social y una sociedad democrática, ayudando a comprender las bases sociales y científicas del trabajo. Esta visión promueve los ideales de John Dewey, quien rechaza la imagen de los estudiantes como individuos pasivos controlados por las fuerzas de la economía de mercado y limitados en su existencia por sus capacidades intelectuales inherentemente prescritas. En su opinión, los estudiantes son sujetos activos y constructores de conocimiento, que requieren vivir y trabajar por un mundo dinámico de bienestar social (Dow, 2002).

La tercera perspectiva critica de manera explícita los dos enfoques anteriores, sugiriendo que la educación técnica por medio de la diferenciación curricular perpetúa las inequidades socioculturales, limitando la movilidad social de las clases trabajadoras. Esto porque desde el sistema escolar los individuos son ordenados y seleccionados para futuros roles ocupacionales en función de sus orígenes de clase. Para esta corriente, los fondos públicos destinados a las escuelas técnicas son la forma más barata de producir trabajadores entrenados sensibles a los valores capitalistas del trabajo y con respeto infundido para el trabajo manual. La educación técnica forma individuos disciplinados que admiten sin alegatos el lugar que se les ha conferido en la estructura de la división del trabajo (Lakes, 1997). 


\section{La educación técnica a inicios del siglo XX}

A inicios del siglo pasado la Asociación Nacional de Manufactureros jugó un rol clave en la instalación de la formación técnica en las escuelas públicas en Estados Unidos. Para este gremio la división del trabajo en las fábricas había hecho impracticable la formación de trabajadores en las empresas, además del hecho de que la introducción de tecnología en los procesos productivos demandaba de nuevas habilidades que solo podían ser adquiridas en escuelas vocacionales especializadas (Kantor, 1986). Sus presiones tuvieron eco en el gobierno federal, que en 1917 y mediante la Ley de Educación Vocacional Smith-Hughes, aprobó el financiamiento de la educación vocacional para jóvenes mayores a 14 años matriculados en escuelas públicas.

Si bien existió consenso en los reformadores americanos acerca de la necesidad de establecer la educación técnica como una alternativa a la clásica tradición académica, había desacuerdo respecto de su diseño específico de implementación. Las dos figuras que representaron los puntos de vista opuestos fueron David Snedden y John Dewey. Para el primero, la formación en escuelas separadas y focalizadas que pudieran reproducir las condiciones de división de trabajo de las fábricas era la mejor forma de preparar para el mundo del trabajo a los jóvenes quienes, debido a sus condiciones intelectuales, no seguían la ruta académica. En cambio, para Dewey la educación vocacional debía ser incluida como parte integral de un currículum comprehensivo que ayudara a los estudiantes a desarrollar un amplio rango de capacidades personales y que acrecentara, más que limitar, sus perspectivas laborales (Hyslop-Margison, 2000; Dow, 2002).

Finalmente, la Ley Smith-Hughes favoreció la visión de Snedden al financiar exclusivamente programas vocacionales que cumplieran con la regla 50-25-25 (50\% en talleres de trabajo, 25\% en contenidos relacionados y $25 \%$ en cursos académicos) y salarios de docentes con experiencia vocacional y no académica. Con ello, tanto los estudiantes como los docentes fueron segregados de sus pares de la vertiente académica, al mismo tiempo que se apartó a la educación técnica al prescribir programas específicos de agricultura, 
comercio e industria y economía doméstica, entre otros (Hayward \& Benson, 1993).

A principios del siglo XX la educación técnica en Chile era conocida como "instrucción especial", e incluía a las escuelas que impartían enseñanza industrial, comercial, agrícola y minera, así como también a la Escuela de Artes y Oficios y a las Escuelas Naval y Militar, entre otras (Soto, 2000). Sin embargo, sus orígenes se remontan a los primeros años del siglo XIX cuando las escuelas de las principales ramas económicas nacen separadamente al alero de sus respectivas asociaciones de productores y en respuesta a la necesidad de contar con mano de obra capacitada para las firmas productoras de la época (Soto, 2000; Dittborn, 2007). Antes, el proyecto fundacional del Instituto Nacional de 1811 que establecía como objetivo formar en ciencias y en artes, así como también en las teorías y elementos prácticos de todos los oficios cuya industria fuera ventajosa para la República, había tomado un rumbo distinto: el de instruir solo en educación liberal (Labarca, 1939).

Fuera del gran instituto, la educación técnica se abre paso muy lentamente en medio de la ausencia de planificación estatal y la falta de recursos tanto humanos como financieros. En 1879, si bien el Estado chileno autoriza por primera vez la inversión de fondos fiscales en establecimientos destinados a la instrucción especial, teórica y práctica; las escuelas que impartían educación técnica como norma estaban al margen del financiamiento estatal regular, debiendo financiarse con recursos propios que provenían de la venta de bienes y servicios que realizaban (Soto, 2000).

Para Magendzo (1969), la tendencia de esos tiempos era separar la educación vocacional de la académica impartida en los liceos, porque se consideraba a la primera menos noble y reservada para los hijos de las clases sociales desventajadas. Por su parte, Soto (2000) da cuenta de que el desprecio por el trabajo manual heredado de los españoles, y el bajo desarrollo del aparato productivo chileno de ese tiempo, están entre los factores culturales y económicos que explican la falta de atención que recibió la educación técnica en sus inicios y hasta muy entrado el siglo XX por parte del Estado. 
Entre 1902 y 1912, en el marco de los congresos nacionales de educación, se produce en Chile una serie de discusiones respecto de la pertinencia de integrar la formación para el trabajo a la educación de nivel secundario. Es así como inspirados en Dewey, educadores como Salas, Galdames y Encina postulan que el fin de la educación es la eficiencia social y que la educación liberal impartida en los liceos no prepara para los potenciales económicos de la vida, sino que más bien los atrofia. Aunque prevalece la noción de que no es misión del liceo entrenar para las carreras de la industria, como fruto del debate se acentúa el rumbo práctico del currículum de la educación de élite mediante el denominado "nacionalismo educativo" que conectó la educación intelectual con la económica (Ruiz, 2010). Pero la educación técnica es ajena a este debate y, al igual que lo que sucede con la educación vocacional segmentada que prevalecía en Estados Unidos a principios del mismo siglo, permanece desligada de la educación académica, pretendiendo formar en cada una de sus ramas un sistema aparte.

\section{Los primeros intentos fallidos de integración}

En los años posteriores a la década de los veinte y hasta mediados de los sesenta no se materializaron cambios significativos en la educación técnica chilena ni en la norteamericana. Sin embargo, importantes debates e intentos frustrados de modificar el nexo de esta educación con la vertiente académica acontecieron en ambos países.

En Estados Unidos, la Asociación Nacional de Educación (NEA) lanzó en 1943 un plan de formación profesional para preparar a los estudiantes de la secundaria para los requerimientos del mercado laboral. El plan incluía un componente de experiencia laboral supervisado para facilitar la transición escuela-trabajo, al mismo tiempo que mantenía una amplia formación general de base para asegurar la capacidad de respuesta de los estudiantes a los cambios de las condiciones locales. A diferencia de la propuesta de Snedden, en el plan de la NEA los estudiantes secundarios no estaban separados en categorías de formación académica y vocacional, sino que seguían programas flexibles e interrelacionados. Esto suscitó el rechazo de los educadores tradicionales, quienes condenaron cualquier intento de 
diluir el contenido académico con la formación vocacional. El plan de transformación de la educación secundaria fue rechazado bajo la premisa de que no se debía permitir a los jóvenes de menos de 17 años con crédito académico tomar cursos vocacionales que paralizaban su desarrollo intelectual (Hyslop-Margison, 2000).

Poco más de una década antes en Chile, otro intento de integrar la educación académica con la vocacional había fracasado, pero no precisamente por razones filosóficas, sino más bien económicas y políticas. En 1928, un decreto supremo estableció la necesidad de crear un sistema unificado de educación secundaria, eliminando la separación existente entre una modalidad que conducía a los estudios superiores y otra al trabajo. Todos los establecimientos educacionales prepararían para ambos objetivos, para lo cual los liceos debían crear secciones técnico-manuales, y los establecimientos vocacionales integrar la formación general. Se pretendía consolidar en el tiempo toda la educación secundaria en una reforma radical cuyo objetivo principal era el de adaptar y orientar la enseñanza a las necesidades de producción y crecimiento del país. Se trataba de una medida que significaba un progreso considerable sobre la estructura anterior, pero su implementación precipitada se mezcló con trabas de orden político y administrativo que finalmente llevaron a abortarla (Labarca, 1939). Para Magendzo (1969) fue una reforma demasiado idealista, cuya magnitud de cambios no era proporcional a las condiciones socioeconómicas y recursos humanos de la época.

En los años siguientes, distintas modificaciones de orden curricular y administrativo acontecen en la educación técnica que pasó a ser llamada "educación vocacional". Entre las más relevantes están la disminución de su duración de seis a cinco años, con lo que sus estudiantes quedaron definitivamente excluidos de alcanzar mayores niveles de escolaridad; $y$, en el plano administrativo, la unificación organizacional de las escuelas vocacionales bajo el mando del Ministerio de Educación que sucede en 1944 (Arnold $\&$ Krammenschneider, 1999). En este periodo se fundan también escuelas de artesanos (carpintería, mecánica, hotelería, textil y artes gráficas, entre otras) destinadas a preparar en determinados oficios y artesanados a jóvenes con educación primaria (Soto, 2000). 


\section{Las reformas estructurales de los sesenta}

En los años sesenta, período en el que la educación técnica en Estados Unidos tuvo un extraordinario crecimiento de matrícula, las preocupaciones por el desempleo juvenil, la decadencia urbana y la disputa de hegemonía mundial con la ex Unión Soviética crearon una crisis en la educación pública de dicho país. Para Hyslop-Margison (2000), las que suscitaron los cambios más relevantes para este tipo de formación desde la Ley Smith-Hughes de 1917 fueron condiciones sociales y económicas ajenas a lo que sucedía en las aulas de las escuelas vocacionales. Antes, la educación vocacional había sido desatendida por reformadores, y dejada en manos exclusivas de sus practicantes. Hayward y Benson (1993) atribuyen el desamparo de la educación técnica norteamericana de esa época, al poco o nulo conocimiento que las autoridades federales y estatales tenían respecto de este tipo de educación, así como también a la falta de interés de la comunidad académica por abordar temas asociados a la educación vocacional.

Los cambios llegaron con la Ley de Educación Vocacional de 1963, la que expandió el alcance y la influencia de esta educación en las escuelas, al destinar fondos específicos para poblaciones desventajadas y eliminar la restricción para financiar programas integrales que combinaran la formación vocacional con la general. La ley obligaba a los estados a impartir programas vocacionales especiales para jóvenes con alguna discapacidad o para aquellos que, producto de alguna desventaja académica o socioeconómica, no se encontraran en condiciones de cursar con éxito programas de educación vocacional regular (Wonacott, 2003). En suma, la nueva legislación norteamericana permitía el financiamiento con recursos federales de cualquier programa profesional de corta duración para mejorar las oportunidades de las personas socioeconómicamente no favorecidas, pudiendo ser estos impartidos en las escuelas secundarias, de adultos, o bien, en los community colleges (Hayward \& Benson, 1993).

Mientras que en el país del norte los programas vocacionales adquirían un matiz social a través de la reforma estructural de 
1963, en Chile la educación técnica que nació considerablemente estratificada era acoplada al sistema escolar, pasando en definitiva a formar parte de la educación media. La reforma educacional del gobierno de Frei Montalva iniciada en 1965 alargó la educación básica a ocho años, y redujo la media a cuatro años y con dos modalidades, científico-humanista y técnico-profesional, agrupando bajo este alero a todas las escuelas vocacionales y de artesanos de la época. Con ello, esta educación pasó a tener el mismo estatus que la educación científico-humanista para efectos de continuidad de estudios, y de transferencia en dichas modalidades de nivel medio (Corvalán y Santibáñez, 1987). Sin embargo, para Ruiz (2010) esta homologación solo ocurrió en términos legales, porque en las orientaciones conceptuales de los reformistas prevalecía la noción de que una modalidad se conectaba con la educación superior, y la otra con el mercado del trabajo.

Por otra parte, a diferencia de la declaración explícita que la legislación norteamericana de 1963 hacía respecto del público objetivo de los programas de educación vocacional especial, en Chile la reforma estructural de los sesenta no alude al tipo de población al que se dirige esta formación. Más bien, desde sus inicios, la opción entre científico-humanista y técnico-profesional es planteada en toda su extensión en términos de preferencias estrictamente individuales.

Para Ruiz (2010) se prescinde en la reforma de Frei Montalva de una discusión más amplia respecto de la relación entre elección del tipo de educación y pertenencia a grupos sociales determinados, que son el foco de las teorías de reproducción social, que conciben a la educación técnica como una perpetuadora de las inequidades socioculturales de las clases trabajadoras. A juicio de esta autora, los cambios que acontecieron en la educación técnica con esta reforma son el resultado indiscutible del movimiento de capital humano de la época y las teorías de la modernización que buscaban una asociación entre educación y desarrollo. 


\section{El declive de la educación técnica norteamericana y la educación técnica chilena en los gobiernos socialista y militar}

Según Castellano, Stringfield y Stone (2003), la osada misión impuesta a la educación vocacional en Estados Unidos de formar a jóvenes en riesgo de desertar del sistema escolar para los puestos laborales requeridos por las industrias, el campo y las oficinas fue cumplida con éxito en la medida en que las plazas de trabajo estuvieron disponibles. Las altas tasas de desempleo que experimentó este país a inicios de los setenta pusieron en jaque a la educación vocacional norteamericana que experimentaba ya cuantiosas bajas en sus tasas de enrolamiento.

En 1974 un estudio nacional identificó una serie de falencias en los programas destinados a poblaciones juveniles vulnerables, los resultados tuvieron repercusión y dos años más tarde los fondos federales otorgados a los estados por la legislación de 1963 fueron drásticamente reducidos con devastadoras consecuencias para la educación vocacional a nivel local. Junto con ello, el Congreso dispuso para la educación vocacional una evaluación nacional cada cinco años (National Assessment of Vocational Education, NAVE) para examinar cuál era la extensión en el cumplimiento del mandato del gobierno federal al respecto.

Los resultados del NAVE no fueron alentadores y el Congreso respondió instituyendo en 1984 la Ley Carl D. Perkins Vocational Education and Applied Technology, con dos objetivos relacionados entre sí, uno económico y otro social. El económico apuntaba a mejorar las habilidades de la fuerza de trabajo y contribuir a la productividad del país, al igual que lo que mandataba la Ley Smith-Hughes de 1917. El objetivo social, en tanto, buscaba proporcionar igualdad de oportunidades a las poblaciones desventajadas mediante la educación vocacional. Según Hayward \& Benson (1993), las legislaciones federales tenían la potencialidad de alinear los esfuerzos estatales en educación vocacional, pero los objetivos de la ley de 1984 eran demasiado ambiguos, al mismo tiempo que pretendían abordar desafíos que requerían de la transformación de todo el sistema 
educativo norteamericano, o al menos los del nivel secundario, y no solo del mejoramiento de la educación vocacional.

En los años sesenta y setenta la educación técnica en Chile, contrariamente al declive que experimentaba la norteamericana, se expandía de forma notable en el sistema escolar. Entre 1965 y 1973 su matrícula se incrementó de 37.400 estudiantes a más de 163.000 (PIIE, 1984, citado por Corvalán y Santibáñez, 1987). Para Núñez (2003) este apogeo de la educación técnica en términos de cobertura fue el resultado de medidas intencionadas del gobierno del presidente Allende, quien, al advertir que hacia fines de 1976 -año de elecciones- el ritmo de aumento de la demanda de educación superior haría colapsar las finanzas públicas, optó por fomentar el crecimiento de la modalidad técnico-profesional de nivel medio para canalizar con ello, voluntariamente, contingentes de alumnos hacia el mercado del trabajo y desviarlos de la educación superior. Según este autor, un régimen con orientación socialista como el de Allende no podía establecer una política de restricción del ingreso, ni tampoco instaurar el pago de aranceles como medida para racionalizar la demanda por estudios superiores, por lo que recurrió a la apertura de liceos técnicos en zonas urbanas. Fueron, entonces, razones políticas y no necesariamente económicas -como mayor demanda del sector productivo o de servicios- las que provocaron el ascenso de la educación técnica en los años setenta.

Pero el gobierno allendista, inspirado en el enfoque crítico de las teorías de reproducción, llevaba arraigada la aspiración de la unidad del sistema escolar chileno en respuesta al problema de segmentación social y al dilema del carácter de la educación secundaria y su relación con las exigencias del desarrollo económico y social. Por ello propuso la Escuela Nacional Unificada (ENU), que contemplaba la ampliación de la educación general en la enseñanza media, pretendiendo superar la división entre trabajo intelectual y trabajo productivo, al integrar las dos modalidades en que aquella se diversificaba en una única educación secundaria con carácter simultáneamente teórico, tecnológico, práctico y manual (Núñez, 2003). El proyecto de la ENU fue resistido con energía en una lucha político-ideológica, por lo que el gobierno del presidente Allende, en un contexto de caos social, no 
insistió en su implementación. Según lo relata Yáñez (2012), entre los argumentos de los oponentes a la ENU estaba el que apuntaba a que detrás de una aspiración falaz de pluralismo, el proyecto de unificación pretendía convertir a la educación chilena en un instrumento de concientización política al servicio del marxismo, confundiendo el concepto de educación con el de adoctrinamiento.

En los años siguientes, durante el régimen militar que sobrevino al gobierno socialista, a pesar de los dramáticos cambios que trajo a la educación técnica el traspaso de sus liceos a los gobiernos municipales, o bien, a corporaciones privadas, esta educación siguió siendo prácticamente la misma que la concebida por la reforma de 1965. Esto a excepción de la libertad de definición de sus programas curriculares que ganaron los liceos técnicos, al terminarse con la obligación que estos tenían de ofrecer solo los mandatados por el Ministerio de Educación (Corvalán y Santibáñez, 1987). A partir del análisis de tareas observadas en puestos de trabajo de empresas de su entorno geográfico, los liceos podían definir autónomamente su oferta de especialidades y sus respectivos planes y programas, poniendo énfasis en que estos se ajustaran a sus demandas y necesidades (Castro y Orellana, 2010).

\section{El cambio de enfoque de la educación técnica norteamericana}

En los años ochenta, tanto en Chile como en Estados Unidos prevalecía en la práctica el paradigma de la eficiencia social que concebía a la educación técnica como una formación especializada que culminaba con la inserción en el mercado laboral, mientras que la vía académica conducía a la universidad. En los dos países, hasta ese momento, ninguna iniciativa de reforma con intenciones integracionistas había obtenido el apoyo popular y político generalizado para concretarse. Eso en Estados Unidos hasta 1990, año en el que una nueva versión de la Ley Carl D. Perkins Vocational Education and Applied Technology, denominada Perkins II, en respuesta a una serie de reportes que acusaban a esta educación de estrechez curricular, deficiente formación general y obsolescencia tecnológica, provocó el más drástico y decidor cambio para la educación vocacional norteamericana (Wonacott, 2003). 
La Ley Perkins II eliminaba formalmente la distinción entre planes de estudio académicos y vocacionales en la secundaria. Por primera vez la legislación de la educación técnica norteamericana se dirigía a todos los segmentos de la población escolar, dejando de fraccionar fondos para estudiantes desfavorecidos y otros para aumentar la calidad de cursos vocacionales regulares. Junto con ello se promovía la articulación de la educación vocacional secundaria con el nivel posecundario, se promocionaban las experiencias formativas en los lugares de trabajo y se establecía la rendición de cuenta como exigencia del financiamiento federal. Como expresión de esta nueva filosofía y el tipo de programas ofrecidos, el término de "educación vocacional" fue cambiado en 1998 por el Career and Technical Education (CTE) (Hayward \& Benson, 1993; Castellano et al., 2003).

Para Dow (2002), fueron los ideales de Dewey que no habían sido totalmente eclipsados por la visión eficientista de Snedden de principios de siglo y las incertidumbres económicas de la década de los setenta los que provocaron un cambio de paradigma en la educación técnica norteamericana. Un nuevo paradigma que alejaba esta educación de las categorías laborales tradicionales y abogaba por una enseñanza más liberal, consistente con los principios democráticos de Dewey y su noción de educación mediante el trabajo, contraria a la de entrenamiento específico.

Las legislaciones posteriores a la de 1990, Perkins III (1998) y Perkins IV (2006) continuaron poniendo énfasis en mejorar la formación académica y la preparación de los jóvenes para la educación posecundaria y el trabajo. Esto con el financiamiento de una serie de manifestaciones de enseñanza y aprendizaje de la CTE en las escuelas secundarias, tales como los programas Tech prep, Career academies, Work-bases youth apprenticeship, Cooperative education, School-based enterprised, entre otros (Rojewski, 2009). Asimismo, estas legislaciones insistían en la rendición de cuentas de los estados, por medio de indicadores clave de rendimiento de la CTE, como aquellos referidos al cumplimiento de estándares de competencias académicas y técnicas, obtención de diplomas de la escuela secundaria o credenciales posecundarias y colocación de egresados en puestos laborales. En particular, la Ley Perkins IV pone un énfasis renovado 
en la evaluación de competencias específicas para una ocupación, o grupo de ocupaciones de los programas de la educación profesional y técnica, y la información que proporciona dicha evaluación (ACTE $\&$ Brustein, 2006).

\section{La educación técnica en Chile en los noventa}

En la primera mitad de los noventa, con la vuelta de la democracia en Chile, se implementó el Programa de Habilitación de Liceos CientíficoHumanistas con Especialidades Técnico-Profesionales (Mineduc, 1992). El programa reunió a las dos modalidades de educación media bajo una misma administración y en la misma infraestructura física, pero no se trató de una apuesta por la integración de la educación media, sino más bien de una cuestión pragmática. A principios de los noventa las altas tasas de desempleo juvenil daban cuenta de la necesidad de vincular a la educación con el trabajo, al menos para los sectores más populares. No se justificaba la creación de nuevos liceos técnicos si existían ya liceos con formación científico-humanista interesados en diversificar su acción educativa. Se optó, entonces, por intervenir estos últimos, decisión que según algunos autores se tomó sin contar con evidencia suficiente que respaldara las bondades de la educación técnica para el mejoramiento de las oportunidades laborales de los jóvenes (Bobenrieth \& Cáceres, 1994; Bellei, 1999).

Como resultado del programa, la matrícula de la educación técnica continuó con su tendencia al alza, alcanzando hacia fines de la década más del $40 \%$ de la matrícula de enseñanza media. Pero el crecimiento había sido caótico, debido a la autonomía con la que contaban los liceos para definir sus propios programas formativos, existiendo más de 400 ofertas distintas de especialidades técnicas en el sistema escolar. La masificación trajo consigo una disminución de calidad que se expresaba fundamentalmente en la baja y desactualizada formación técnica y pedagógica de los docentes de especialidad, así como también en la desvinculación de los liceos con el mundo productivo (Miranda, 2003).

Con el crecimiento de la matrícula de educación técnica el nivel de enseñanza media pasó a transformarse de selectivo a masivo, 
pero con vacíos de diagnóstico y con visiones encontradas acerca de su desarrollo futuro. Para hacer frente a esta situación el Ministerio de Educación encargó varios estudios con el propósito de establecer bases de conocimiento adecuadas para el diseño de una política de renovación de la educación media (Lemaitre, Cerri, Cox \& Rovira, 2003). Los resultados de los estudios dan cuenta de discusiones académicas y políticas amparadas por organismos internacionales respecto de la pertinencia de retrasar o incluso postergar al nivel posecundario la separación entre educación técnica y académica.

La Reforma Curricular de la Educación Media de 1998, alineada con la tendencia internacional, pospone para el último tramo de este nivel educativo ( $3^{\circ}$ y $4^{\circ}$ medio) la separación entre las modalidades científico-humanista y técnico-profesional. Junto con ello, reorganiza su oferta formativa en 14 sectores económicos y 46 vías de especialización, cuyos perfiles de egreso contenidos en el marco curricular son mandatorios para todos los establecimientos que imparten este tipo de educación. Finalmente, la reforma insiste en que la educación técnico-profesional prepara tanto para el ingreso al mercado laboral como para la continuidad de estudios superiores. Sin embargo, no avanza en promover puentes formales que faciliten el tránsito de los egresados de la educación técnica hacia la educación superior.

\section{La educación técnica en el siglo XXI}

En Chile, las iniciativas recientes que ponen el foco en la educación técnica, como es el caso del Programa Chilecalifica (2002-2008), que pretendió crear un sistema de formación permanente (Mineduc, 2006), el plan de equipamiento de liceos técnicos (2010-2013) y las nuevas bases curriculares (2013) (Mineduc, 2014), se concentran en mejorar y fortalecer el sistema existente. Después de la reforma de 1998 no acontecieron cambios relevantes para esta educación, ni para el nexo que esta tiene con la vía científico-humanista. No se produce en nuestro país un debate respecto de la pertinencia de mantener la estructura del sistema vigente o establecer uno alternativo, con excepción de la constitución, en el año 2008, de una comisión externa para revisar los antecedentes disponibles acerca de la educación 
técnico-profesional y la elaboración de propuestas para fortalecerla, que se tradujo en un informe ejecutivo sin repercusiones mayores.

El sistema actual promete una formación tanto para una inserción laboral temprana como para la continuidad de estudios superiores. Sin embargo, esta misión es incongruente en su diseño curricular, ya que no contempla nexos formales con el nivel terciario. En un escenario de expansión de la educación superior y de mayor inversión pública en ella, el discurso de la educación técnica del sistema escolar como modalidad no terminal y válida para la continuidad de estudios solo puede sostenerse en la medida en que este tipo de educación fortalezca sus contenidos académicos y se articule con las instituciones que imparten carreras técnicas de nivel superior.

En cambio, Estados Unidos ha sustentado el mandato de sus programas técnicos escolares de proyectar a sus estudiantes al nivel posecundario, en acciones legislativas sostenidas en el tiempo como el Programa Tech Prep y recientemente los Programs of Study (POS). Ambas iniciativas, basadas en la alineación de contenidos técnicos y el reforzamiento de la formación académica, entre otros aspectos, buscan facilitar la progresión coordinada y no duplicada de cursos conducentes a un certificado o título ocupacional de educación superior desde la secundaria (Dortch, 2012).

En particular, para la implementación de los POS, la Asociación Nacional de Directores de la CTE (NASDCTEc) ha debido recurrir a la creación de marcos referenciales de acción para los proveedores de educación vocacional, mediante los National Career Clusters (Grupos de Carrera Nacionales) que proveen una estructura para la organización y provisión de ofertas formativas articuladas. Este dispositivo, que contiene estándares de conocimiento y habilidades para 16 grupos de carreras y más de 70 itinerarios de formación, junto con ser una herramienta para el diseño curricular de los oferentes, pretende constituirse en una guía para que los estudiantes planifiquen sus trayectorias formativas desde la secundaria hacia la educación superior (NASDCTEc, 2014). 


\section{Reflexiones finales}

Aquí se ha relatado una larga selección de hitos de la educación técnica en Chile y en Estados Unidos desde principios del siglo XX hasta los tiempos actuales. Sin embargo, no se trata de una sistematización cerrada de la historia de la educación técnica en ambos países, sino más bien de una reflexión abierta hacia el despegue de una discusión y debate más amplio respecto de la educación técnica en el sistema escolar chileno y su relación con la vertiente académica y la educación superior. Para ello se han descrito también los hechos que marcaron la evolución de la educación técnica en el país del norte. El objetivo fue tener un punto de referencia y contrastación para ampliar la mirada hacia otros contextos, bajo el marco de referencia de los distintos enfoques conceptuales de acercamiento a la educación técnica.

En Estados Unidos, la educación técnica sigue un recorrido marcado por reformas con énfasis en lo económico (Ley SmithHughes de 1917), lo económico y lo social (Ley Educación Vocacional de 1963), y lo académico, económico y social (Ley Carl Perkins de 1990 y sus versiones posteriores). Esto la lleva a evolucionar desde una formación curricularmente restringida y focalizada en responder a las necesidades de la industria, hasta una formación amplia que comprende cualquier experiencia o actividad de aprendizaje que pueda conducir a los alumnos a conseguir competencias válidas para el mercado laboral y la educación superior. Son razones económicas, sociopolíticas e ideológicas las que se suman en el tiempo y hacen que la educación técnica en este país transite desde un enfoque técnicofuncionalista, hasta otro integracionista que aboga por la eliminación de barreras entre la educación vocacional y académica.

En Chile, en cambio, si bien la educación técnica en el transcurso del tiempo presenta varios procesos de acoplamiento a la educación académica que se constituyen en ejes ordenadores que marcan su evolución histórica (reformas de 1965 y 1998 fundamentalmente), nunca se produce un cambio de paradigma, o al menos los modelos conceptuales más sociales fallan en ser implementados. El enfoque técnico-funcionalista que mira a la educación técnica como una formación especializada en función de los requerimientos del aparato 
productivo es el que desde siempre domina las orientaciones políticas para esta educación.

Pero la implementación de modelos integracionistas en el nivel secundario no es la panacea para la educación técnica. En Estados Unidos la Career and Technical Education no está ajena a críticas, así como tampoco lo están los programas vocacionales del sistema escolar de Australia, por nombrar otro ejemplo. En este caso, el total ajuste de la educación técnica en las escuelas comprehensivas australianas es cuestionado por quienes claman por el mantenimiento de la identidad de este tipo de educación, así como también por el reconocimiento de la opción de los jóvenes que no desean continuar estudios posecundarios y que más bien quieren trabajar (Polesel \& Clarke, 2011). Mientras que en el país del norte se acusa a las escuelas secundarias comprehensivas y aquellas sin distinción entre planes académicos y vocacionales de, en primer lugar, estar resueltamente orientadas a preparar a todos sus estudiantes para el college, sin reconocer que el $60 \%$ de sus egresados nunca lo concluye; y, en segundo lugar, de tampoco contar con una formación específica consolidada que les permita realizar una actividad laboral con algún grado de calificación (Stone \& Lewis, 2012).

Se requiere, entonces, de un debate amplio en torno a la situación de la educación técnica en el sistema escolar chileno, que la aborde a partir de su nexo con la formación general y sus proyecciones hacia la educación superior, sin olvidar su carácter profesionalizante orientado al mercado del trabajo. Solo una mirada sistémica y desde distintos enfoques conceptuales permitirá desarrollar políticas integrales de largo plazo que se traduzcan en mejores oportunidades para los jóvenes a quienes convoca este tipo de educación. Dentro de estas políticas se rescata la imperiosa necesidad que tiene el sistema educativo chileno de avanzar en la creación de un marco nacional de cualificaciones que, junto con establecer itinerarios formativos entre distintos niveles, propicie el alineamiento de la oferta formativa con los requerimientos del sector productivo. 


\section{Referencias}

ACTE, \& Brustein, M. (2006). The Perkins Act 2006 - The Official Guide. Alexandria, Virginia: ACTE.

Arnold, R. \& Krammenschneider, U. (1999). Caught between private and public interests: vocational training in Chile. International Journal of Sociology, 29(2), 5-28.

Bellei, C. (1999). Equidad social y expansión de la educación media técnicoprofesional, un estudio de caso, en Proposiciones, 27, Santiago de Chile: Ediciones SUR, 1999. Recuperado el día 30/03/2014 desde http:// www.sitiosur.cl/r.php?id=635.

Benavot, A. (1983). The rise and decline of vocational education. Sociology of Education, 56(2), 63-76.

Bobenrieth, E. \& Cáceres, C. (1994). Un modelo de selección de liceos de enseñanza media. Cuadernos de Economía, 31(92), 27-44.

Castellano, M., Stringfield, S., \& Stone, J. (2003). Secondary career and technical education and comprehensive school reform: implications for research and practice. Review of Educational Research, 73(2), 231-272.

Castro E., \& Orellana, W. (2010). La educación media técnico profesional en Chile: entre la desarticulación y la indefinición. Intersecciones educativas. Número 2, 73-81.

Corvalán, O. \& Santibáñez, E. (1987). Transformaciones de la educación técnico-profesional. Balance y perspectivas. Santiago: CIDE (Documento de trabajo, 24).

Dittborn, P. (2007). Historia y perspectivas acerca de la educación técnica de nivel superior. Revista Calidad en la Educación, 27(2), 17-33.

Dortch, C. (2012). Carl D. Perkins Career and Technical Education Act of 2006: Implementation Issues. Congresional Research Service. December, 2012.

Dow, J.L. (2002). The new vocationalism: A Deweyan analysis. Doctoral dissertation, December 2002, University of Florida.

Hayward, G.C., \& Benson, C.S. (1993). Vocational-technical education: major reforms and debates 1917- present. Washington, DC: U.S. Department of Education, Office of Vocational and Adult Education.

Hyslop-Margison, E.J. (2000). An assessment of the historical arguments in vocational education reform. Journal of Career and Technical Education, $17(1), 23-30$. 
Kantor, H. (1986). Work, education, and vocational reform: the ideological origins of vocational education, 1890-1920. American Journal of Education, 401-426.

Labarca, A. (1939). Historia de la educación en Chile. Santiago de Chile: Editorial Universitaria.

Lakes, R.D. (1997). New vocationalism: Deweyan, Marxist, and Freirean themes. Retrieved from ERIC database. (ED408508).

Lemaitre, M.J., Cerri, M., Cox, C. \& Rovira, C. (2003). La reforma de la educación media. Políticas educacionales en el cambio de siglo. Santiago de Chile: Editorial Universitaria.

Magendzo, A. (1969). A historical review of the development of vocational education in Chile. Doctoral dissertation, University of California.

Mineduc (1992). Aprueba "Programa de habilitación de establecimientos de enseñanza media humanístico-científica en técnico-profesional. República de Chile, Ministerio de Educación, División de Educación General. Resolución exenta No 1854/92. Santiago, 19 de mayo.

Mineduc (2006). Más y mejores técnicos para Chile. Santiago de Chile: Chilecalifica.

Mineduc (2014). Cuenta Pública de Educación 2010-2014. Recuperado el día 30/03/2014 desde http://www.mineduc.cl//usuarios/mineduc/doc/ cuenta_publica2013_e.pdf

Miranda, M. (2003). Transformación de la educación media técnicoprofesional en políticas educacionales en el cambio de siglo. En Cox, C. (Ed.) Políticas educacionales en el cambio de siglo. La reforma del sistema escolar de Chile (pp. 375-417). Santiago de Chile: Universitaria.

NASDCTEc (2014). Pathways to college \& career readiness. Recuperado el día 30/03/2014 desde http://www.careertech.org/career-clusters/glance/.

Núñez, I. (2003). La ENU entre dos siglos: ensayo histórico sobre la Escuela Nacional Unificada. Santiago: LOM Ediciones.

Polesel, J., \& Clarke, K. (2011). The marginalization of VET in an australian secondary school. Journal of Vocational Education \& Training, 63(4), 525-538.

Rojewski, J. W. (2009). A conceptual framework for technical and vocational education and Training. In International Handbook of Education for the Changing World of Work (pp. 19-39). Netherlands: Springer.

Ruiz, C. (2010). De la República al mercado, ideas educacionales y política en Chile. Santiago: LOM Ediciones. 
Soto, F. (2000). Historia de la educación chilena. Santiago: CPEIP, Ministerio de Educación.

Stone, J., \& Lewis, M. (2012). College and career ready in the 21st Century: making High School Matter. New York, N.Y.: Teachers College Press.

Yáñez, J.P. (2012). La ENU como representación de la lucha político-ideológica durante la Unidad Popular. Revista Divergencia, 1(1), 73-94.

Wonacott, M.E. (2003). History and evolution of vocational and career-technical education. A compilation. Columbus, $\mathrm{OH}$ : Center on Education and Training for Employment.

Recibido: 31/03/2014 Aceptado: 28/04/2014 